\title{
An analysis on the determinants of head to pelvic balance in a Chinese adult population
}

\author{
Zongshan $\mathrm{Hu}^{1,2,3 \#}$, Claudio Vergari ${ }^{4 \#}$, Laurent Gajny ${ }^{4}$, Gene Chi-Wai Man ${ }^{2,3}$, Kwong-Hang Yeung ${ }^{3,5}$, \\ Zhen Liu ${ }^{1}$, Tsz-Ping Lam ${ }^{2,3}$, Zezhang Zhu ${ }^{1,3}$, Yong Qiu, ${ }^{1,3}$, Winnie Chiu-Wing Chu ${ }^{3,5}$, \\ Jack Chun-Yiu Cheng ${ }^{2,3}$, Wafa Skalli ${ }^{4}$
}

${ }^{1}$ Department of Spine Surgery, Drum Tower Hospital of Nanjing University Medical School, Nanjing, China; ${ }^{2}$ Department of Orthopaedics and Traumatology, The Prince of Wales Hospital, The Chinese University of Hong Kong, Hong Kong, China; ${ }^{3}$ The Joint Scoliosis Research Center of the Chinese University of Hong Kong-Nanjing University, Faculty of Medicine, The Chinese University of Hong Kong Hong Kong, China; ${ }^{4}$ Arts et Métiers Institute of Technology, Université Sorbonne Paris Nord, IBHGC-Institut de Biomécanique Humaine Georges Charpak, HESAM Université, F-75013, Paris, France; ${ }^{5}$ Department of Imaging and Interventional Radiology, The Prince of Wales Hospital, The Chinese University of Hong Kong, Hong Kong, China

Contributions: (I) Conception and design: W Skalli; (II) Administrative support: JCY Cheng; (III) Provision of study materials or patients: GCW Man, KH Yeung, TP Lam, WCW Chu; (IV) Collection and assembly of data: Z Hu, C Vergari, L Gajny; (V) Data analysis and interpretation: Z Hu, C Vergari, Z Liu, Z Zhu, Y Qiu; (VI) Manuscript writing: All authors; (VII) Final approval of manuscript: All authors.

"These authors contributed equally to this work.

Correspondence to: Jack Chun-Yiu Cheng. Department of Orthopaedics and Traumatology, Faculty of Medicine, The Chinese University of Hong Kong, The Prince of Wales Hospital, Shatin, Hong Kong, China. Email: jackcheng@cuhk.edu.hk; Wafa Skalli. Arts et Metiers ParisTech, Institut de Biomecanique Humaine Georges Charpak, 151, Boulevard de l'Hôpital 75013 Paris, France. Email: wafa.skalli@ensam.eu.

Background: Balanced global spinopelvic balance is important in the maintenance of the physiological alignment of all body segments above the pelvis with minimum energy expenditure. The key determinants affecting the 3D index-odontoid-hip axis (OD-HA) angle, and in particular its alterations, have not been clearly defined. The aim of this study is to identify the determinants of the 3D OD-HA angle in maintaining global spinopelvic balance in a large Chinese adult cohort of different gender and age groups.

Methods: A total of 516 asymptomatic adults were enrolled in this study. Biplanar radiographies were performed to reconstruct the subject's inter-acetabular axis and C2 odontoid process. The 3D angle formed by the vertical and the line between odontoid and mid-interacetabular axis (OD-HA angle) was computed and projected in the subject's sagittal and coronal planes. Thoracic kyphosis (TK), lumbar lordosis (LL), T1 pelvic angle (TPA) and sagittal vertical axis (SVA) were measured.

Results: The mean values of sagittal and coronal OD-HA were $-0.2^{\circ} \pm 2.5^{\circ}$ and $0.2^{\circ} \pm 1.1^{\circ}$, respectively. Both sagittal and coronal OD-HA had significant correlation with age $(\mathrm{r}=0.265$ and $\mathrm{r}=-0.143, \mathrm{P}<0.01$, respectively), sagittal OD-HA showed increment from $20 \mathrm{~s}$ to $80 \mathrm{~s}\left(-1.3^{\circ}\right.$ to $0.8^{\circ}$ for female, $-0.3^{\circ}$ to $1.5^{\circ}$ for male) and a significant difference between male and female from 20 to 69 years old. Further analysis showed that sex, weight, TK, PT, SVA, TPA and ODI were determinants of OD-HA.

Conclusions: 3D OD-HA angle showed physiological stability with little variability from young to elderly adults, with $\mathrm{SD}$ of $2.45^{\circ}$ and $1.06^{\circ}$ in sagittal and coronal planes, respectively. OD-HA angle confirms the hypothesis that the head tends to remain above the pelvis in a small cone of stability. This study provides an analysis of the determinants of OD-HA and the reference range of the head-pelvis balance in each decade and gender based on a large-scale asymptomatic population.

Keywords: 3D reconstruction; asymptomatic; adult; postural alignment; odontoid-hip axis (OD-HA) 
Submitted Jul 11, 2021. Accepted for publication Dec 16, 2021 ; Published online: 07 Jan 2022.

doi: 10.21037/qims-21-718

View this article at: https://dx.doi.org/10.21037/qims-21-718

\section{Introduction}

"Global spinal balance" was considered as the overall alignment of spine, usually by taking centroid of C7 vertebral body and the sacrum or pelvis as the upper and lower reference points (1). The concept of "conus of economy" by Dubousset suggested the presence of a narrow physiological range within which the human body can remain globally balanced three-dimensionally (3D) (2).

Over the past decades, growing emphasis on the significance of assessing radiographic images in the sagittal plane and its correlation with clinical pain and disability was observed $(3,4)$. However, these clinical findings were mostly based solely on 2D images. A variety of methods have been developed in trying to evaluate the $3 \mathrm{D}$ spinal deformity, e.g., basing on the projection of the pedicles or spinous process on coronal $\mathrm{X}$-ray (5). In an effort to characterize the axial plane, the radiographic evaluation of adult spinal deformity (ASD) patients is often complemented by CT or MRI scan with its intrinsic limitation of having the image acquisition in supine and non-weight-bearing position (6). This often led to inadequate estimation of the true $3 \mathrm{D}$ deformity that could correlate reliably with the clinical pain or functional impairments $(7,8)$. In addition, the radiation exposure during CT scan acquisition is a concern particularly in longitudinal investigations. To overcome these obstacles, low-dose whole-body biplanar imaging system was introduced and became increasingly available in clinical practice to allow imaging from head to toe in standing position without projection distortions. Likewise, with a dedicated algorithm, these biplanar images can be processed to generate an accurate $3 \mathrm{D}$ reconstruction for further analysis in three anatomical planes $(9,10)$.

There is growing recognition of the importance of a balanced global spinal balance in maintaining the proper physiological alignment of all body segments above and below the pelvis thus providing the least energy demanding standing posture. The failure to maintain the center of gravity in this "conus of economy" would require a compensatory mechanism to restore a stable global spinal balance. In addition, the weight of the head estimated to be 4 to $5 \mathrm{~kg}$ in adults is supported by the flexible cervical spine, followed by the thoracolumbar construct (11). Its spatial position is thus of great importance in the global spinal balance. Making full use of the potential of EOS imaging system, Amabile et al. proposed a new 3D parameter: the odontoid-hip axis (OD-HA) angle to quantify the 3D headpelvis alignment in young asymptomatic adults (12). Further studies showed that this parameter was quasi-invariant in a large part of the population, including older adults, both Caucasian and Chinese, and adolescent idiopathic scoliosis patients before and after surgery (13-15).

Previous studies have described the progressive changes of spinopelvic alignment of the normal and pathologic aging spine $(16,17)$. In an effort to maintain the alignment of human body's segments, including head, spine, pelvis, lower limbs, the young subjects have the capacity to regulate their spinopelvic alignment by fine-tuning the curvature of their spine and adjusting the orientation of their pelvis (12). After surgery in adolescent idiopathic scoliosis (AIS), it has been shown that they even a shape of the pelvis can be involved in this adaptation process, since a change of incidence parameter is frequently observed (18). Previous studies on different ethnicities also showed significant differences in the sagittal alignment (19-21). In addition, our recent study has found that sagittal alignment could vary with age and gender, and the elderly had a different compensation mechanism to keep balance beyond which decompensation could occur. However, the factors affecting OD-HA, have not been clearly defined nor analyzed yet, and such information could further clarify the compensation mechanisms deployed by the subject to keep balance. Therefore, the objective of this study was to identify the determinants of the 3D OD-HA angle in maintaining global spinopelvic balance in a large-cohort of Chinese adults of different gender and age groups. We present the following article in accordance with the MDAR checklist and STROBE reporting checklist (available at https://qims.amegroups.com/ article/view/10.21037/qims-21-718/rc).

\section{Methods}

\section{Study design}

This was a cross-sectional study with approval from our institutional Ethics Committee (CREC Ref. No. 2017.689) 

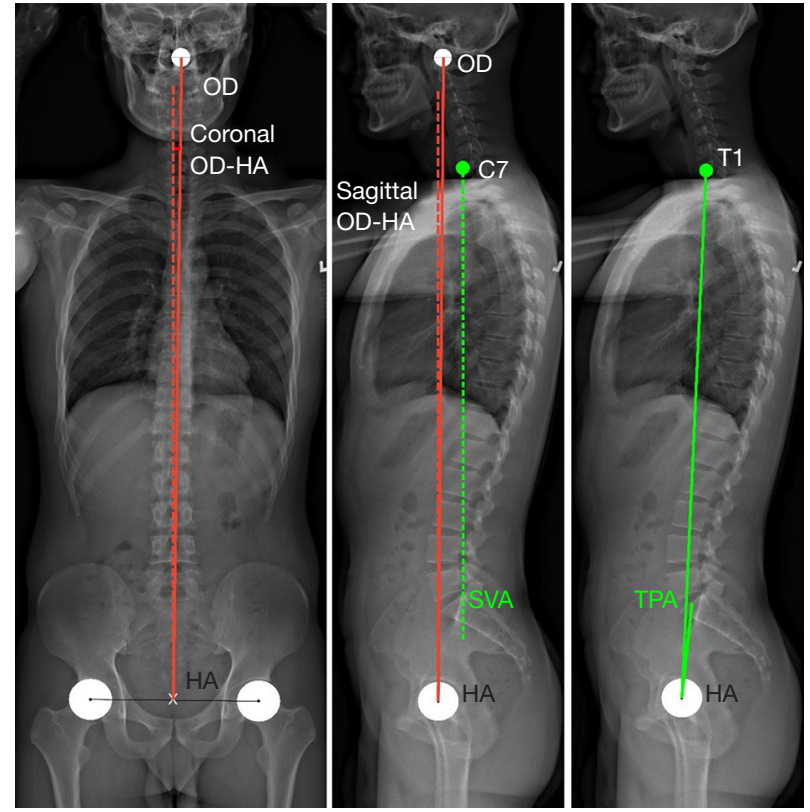

Figure 1 Anteroposterior and lateral X-rays of an asymptomatic adult illustrating the calculation of coronal and sagittal OD-HA angle between the vertical and the line connecting the C2 OD and the midpoint of the HA with a $4^{\circ}$ of TPA and $0.7 \mathrm{~mm}$ of SVA. OD-HA, odontoid-hip axis; TPA, T1 pelvis angle; SVA, sagittal vertical axis.

conducted in the light of the Declaration of Helsinki (as revised in 2013). All volunteers were fully informed about the methods, purposes, and risks involved in the study protocol and signed the informed consent.

\section{Subjects}

Asymptomatic Chinese volunteers' age between 20-89 years old were recruited from the different geographical regions of the community. Bi-planar X-rays were obtained with the EOS system, a low-dose system permitting the simultaneous head to feet acquisition of sagittal and coronal X-rays, between November 2016 and September 2018. Inclusion criteria were subjects presenting an Oswestry disability index (ODI) score lower than $20 \%$ and a VAS score (visual analog scale) lower than $2 \%$ in assessing the back pain (including neck pain). The exclusion criteria were subjects with neck and back pain in previous 6 months, presence of neuromuscular disorders, pregnant, and previous pathology or surgery on spine, hip or knee arthroplasty that could affect coronal or sagittal balance. Basic demographic characteristics of the subjects, including age, body height, body weight and body mass index (BMI), were also recorded.

\section{EOS low-dose biplanar scan}

All subjects underwent whole-body biplanar stereographs (EOS imaging, Paris, France), which allows a simultaneous capture of anteroposterior and lateral radiographs by slot scanning of the whole body in an upright, weight-bearing position with ultralow radiation doses, with a standardized radiographic protocol by a team of experienced radiographers. Subjects were asked to stand up in the standardized free-standing position, adapted from Faro et al. (22), with hands resting on the mandibles (SRS modified free standing position) and with shifted feet. A medical practitioner familiar to EOS radiographs and not involved in the study blindly reviewed all subjects' radiographs to label and reject radiographs of subjects with incorrect posture.

\section{Radiographic measurements and calculation}

Two orthopedic specialists who were not otherwise involved in this study performed all the radiographic measurements, and the average of their results was recorded. The following parameters were measured: (I) global parameters: sagittal vertical axis (SVA) and T1 pelvic angle (TPA). (II) Local curvature: lumbar lordosis (LL) and thoracic kyphosis (TK). (III) The measurements of sagittal parameters are shown in Figure 1.

The 3D OD-HA angle was formed from measuring the position of the most superior tip of the odontoid process of C2 (OD) using a validated technique. The OD-HA angle was defined as the angle between the vertical line crossing the center of the hip axis (HA) and a line between $\mathrm{OD}$ and $\mathrm{HA}$; this angle was measured in $3 \mathrm{D}$ and projected in the anatomical sagittal and coronal planes (Figure 2). MATLAB $^{\circledast}$ was used to automatically compute the values of OD-HA and other radiographic parameters (12).

\section{Statistical analysis}

Data were expressed as mean \pm standard deviation. The measurements were tabulated and analyzed using the SPSS version 26.0 software (SPSS Inc., Chicago, IL, USA) and Matlab (The Mathworks, Natick, USA). To assess the relationship between spinopelvic parameters with coronal and sagittal OD-HA, correlations with all parameters were 

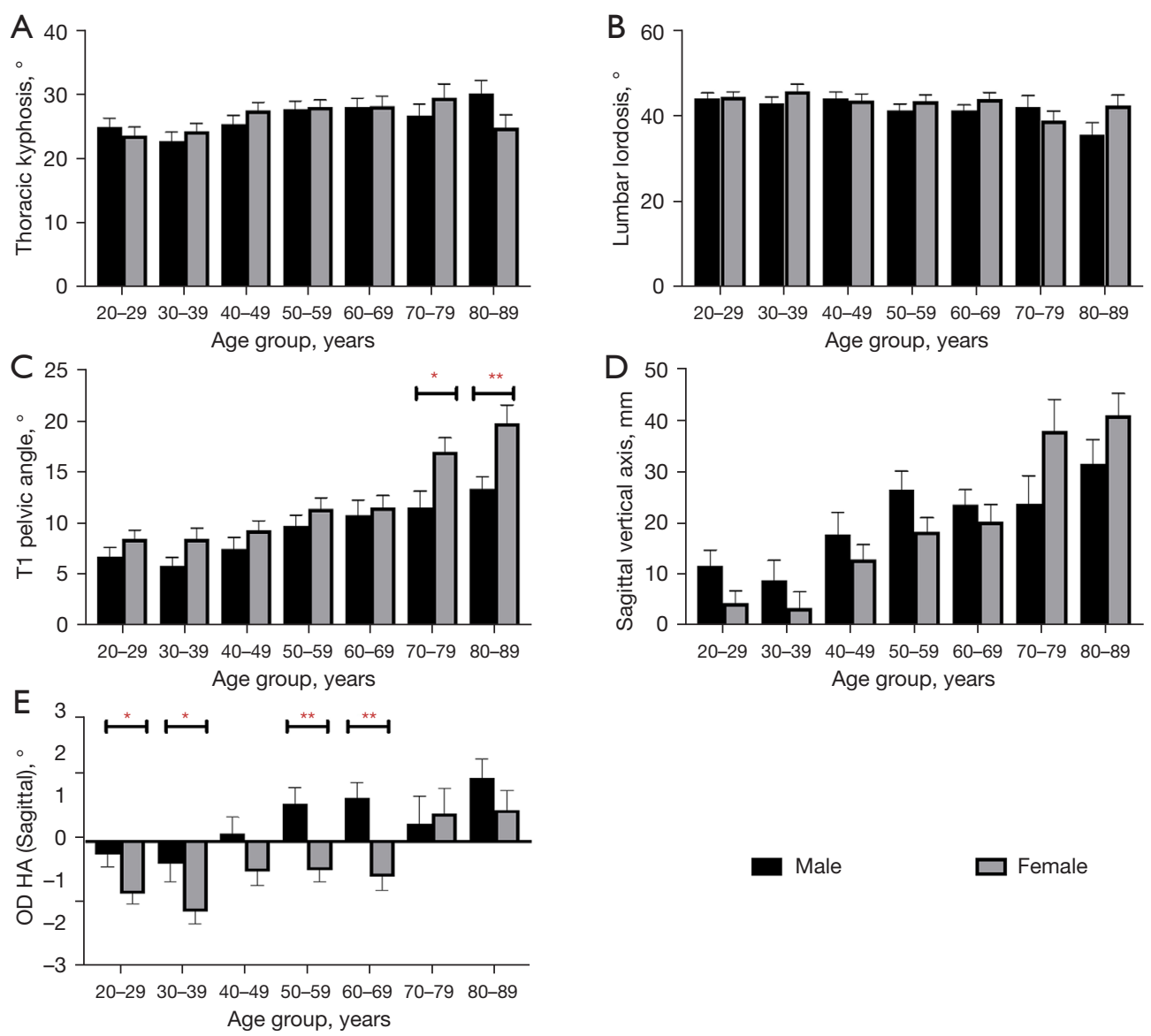

Figure 2 Mean value of whole-body sagittal parameters by age between males and females. * $\mathrm{P}<0.05 ;{ }^{* *}, \mathrm{P}<0.01$.

analyzed. Then, stepwise regression was used to determine which spinopelvic and clinical parameters affect OD-HA; sagittal and coronal projections of this angle were processed separately. Then, a multilinear model was built to determine the relationship between these variables and OD-HA. Finally, a principal component analysis was run to study the relationship between these relevant variables.

Normal distribution of the values was confirmed by Shapiro-Wilk normality test for each series of measurements. Comparisons of means between variables were performed using an unpaired Student $t$-test or Mann-Whitney tests for non-normally distributed variables. Comparisons among age groups were made with analysis of variance. Correlations between variables were analyzed using the Pearson correlation coefficient or Spearman's rank test. The interobserver and intra-observer reliability were assessed with an absolute agreement intraclass correlation coefficient (ICC) analysis using a two-way random effects model. Agreement was classified as excellent for an ICC of $>0.75$. Values of $\mathrm{P}<0.05$ was considered statistically significant.

\section{Results}

\section{Demographic data}

A total of 516 subjects (female to male: 280:236) were successfully recruited with the demographic data distribution and a minimum of 20 subjects of each sex in each age group, as shown in Table 1. The average age of the subjects was 51.9 years (range, 20-89 years) and the BMI was $23.5 \pm 3.5 \mathrm{~kg} / \mathrm{m}^{2}$. The average ODI score and VAS score were $13.4 \pm 12.7$ and $1.8 \pm 2.1$, respectively, reflecting the asymptomatic cohort that matched with the inclusion criteria. The ICC values for the intra- and inter-observer agreement in EOS measurements with the sagittal parameters were higher than 0.9 , indicating reliable measurement methods. 
Table 1 Demographic distribution of study participants

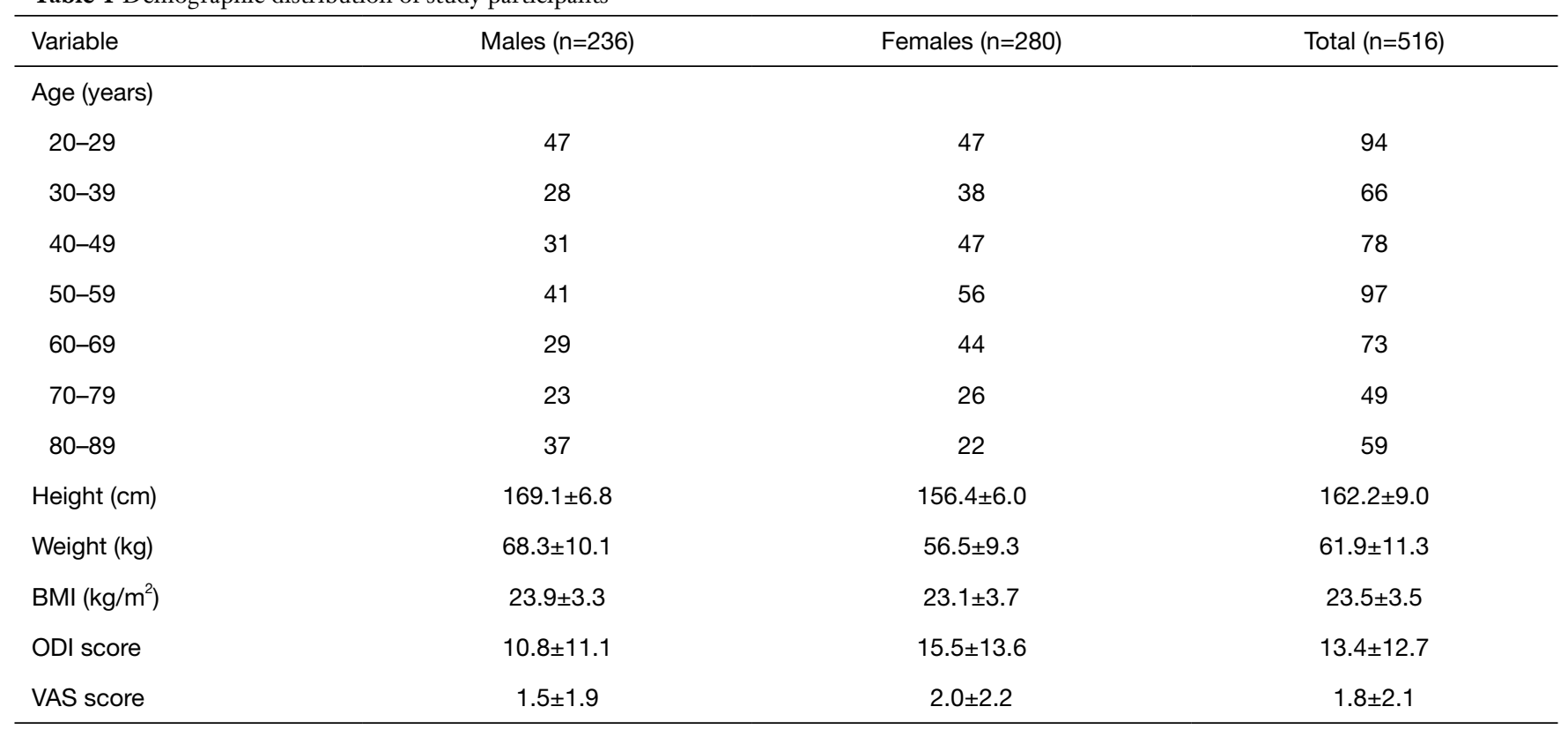

Data are presented as number or mean \pm SD. BMI, body mass index; ODI, Oswestry disability index; VAS, visual analogue scale.

\section{Changes in spinal alignment}

The mean values of sagittal and coronal OD-HA were $-0.17^{\circ} \pm 2.45^{\circ}$ and $0.16^{\circ} \pm 1.06^{\circ}$, respectively. The overall trend for each sagittal variable in males and females by age decade were presented in Figure 2 containing five subparts (Figure 2A-2E). Across the different age groups, TK showed steady increasing trend while LL gradual decrease in both genders (Figure $2 A, 2 B$ ). The global sagittal parameters, TPA and SVA, increased gradually with age from 20 to 89 in both genders (Figure 2C,2D). However, there is a significant higher increase in these parameters on female subjects aged above 70 years old when compared with male subjects $(\mathrm{P}<0.05)$.

\section{OD-HA angle}

The average values and overall trend for OD-HA angle in males and females by decade were shown in Figure 2E. The mean value of sagittal OD-HA angle in female subjects and male subjects were $-0.7^{\circ} \pm 2.3^{\circ}$ and $0.5^{\circ} \pm 2.5^{\circ}$, respectively. Sagittal OD-HA showed significant changes for male $\left(-0.3^{\circ}\right.$ to $\left.1.5^{\circ}\right)$ and female $\left(-1.3^{\circ}\right.$ to $\left.0.8^{\circ}\right)$ subjects from 20 s to 80 s.

The comparison on OD-HA, TPA and SVA between young adults and elderly adults is shown in Table 2. In female subjects, there were significant differences in OD-HA angle $\left(-1.20^{\circ}\right.$ vs. $\left.-0.28^{\circ} ; \mathrm{P}<0.01\right)$, TPA $\left(8.79^{\circ}\right.$ vs. $\left.13.70^{\circ} ; \mathrm{P}<0.01\right)$ and SVA $(7.17 \mathrm{~mm}$ vs. $25.70 \mathrm{~mm} ; \mathrm{P}<0.01)$ when compared between young adults and old adults. Similarly, there were significant differences in OD-HA angle $\left(-0.22^{\circ} v s .1 .04^{\circ}\right)$, TPA $\left(6.73^{\circ} v s .11 .30^{\circ}\right)$ and SVA $(12.68 v s .26 .83 \mathrm{~mm})$ when compared between young adults and old adults in male subjects $(\mathrm{P}<0.05)$.

\section{Correlation between $\mathrm{OD}-\mathrm{HA}$ and other parameters}

A summary of the correlations observed between spinopelvic and clinical variables is shown in Figure 3. Coronal OD-HA only showed a mild negative correlation with age in females $(\mathrm{r}=-0.14, \mathrm{P}=0.02)$, while not in male $(\mathrm{P}>0.05)$. In females, SVA was correlated with age $(r=0.45, \mathrm{P}<0.001)$, but not with ODI and TPA $(\mathrm{P}>0.05)$. However, sagittal OD-HA in this group tended to decrease with subject height and PT ( $\mathrm{r}=-0.2, \mathrm{P}<0.001$ and $\mathrm{r}=-0.14, \mathrm{P}=0.02$, respectively), and to increase with $\mathrm{BMI}(\mathrm{r}=0.16, \mathrm{P}=0.01)$. On the other hand, sagittal OD-HA angle in males showed small but significant increase with age $(\mathrm{r}=0.3, \mathrm{P}<0.0001)$, TPA $(\mathrm{r}=0.2, \mathrm{P}=0.002)$, ODI $(\mathrm{r}=0.15, \mathrm{P}=0.02)$ and VAS $(\mathrm{r}=0.13, \mathrm{P}=0.04)$, and $\mathrm{a}$ strong correlation with SVA $(r=0.8, \mathrm{P}<0.0001)$.

In addition, a stepwise correlation analysis was performed to determine which among the parameters measured in this study were determinant variables for sagittal or coronal 
Table 2 Comparison of global sagittal alignment between young and elderly adults

\begin{tabular}{lcccc}
\hline \multirow{2}{*}{ Variable } & \multicolumn{2}{c}{ Young adults $(<50$ years $)$} & \multicolumn{2}{c}{ Elderly adults $(\geq 50$ years $)$} \\
\cline { 2 - 5 } & Male $(\mathrm{N}=106)$ & Female $(\mathrm{N}=132)$ & Male $(\mathrm{N}=130)$ & Female $(\mathrm{N}=148)$ \\
\hline TK & $24.5 \pm 8.4$ & $25.2 \pm 8.7$ & $28.3 \pm 9.5^{\#}$ & $27.9 \pm 9.9^{*}$ \\
LL & $43.8 \pm 8.3$ & $44.5 \pm 9.8$ & $39.8 \pm 12.4^{\#}$ & $42.7 \pm 11.2$ \\
TPA & $6.7 \pm 5.8$ & $8.8 \pm 6.2$ & $11.3 \pm 7.8^{\#}$ & $13.7 \pm 8.4^{\star *}$ \\
SVA & $12.7 \pm 22.2$ & $7.2 \pm 19.0$ & $26.8 \pm 24.3^{\#}$ & $25.7 \pm 25.2^{\star *}$ \\
OD-HA & $-0.2 \pm 2.2$ & $-1.2 \pm 2.0$ & $1.0 \pm 2.6^{\#}$ & $-0.3 \pm 2.5^{\star *}$ \\
\hline
\end{tabular}

${ }^{*}, \mathrm{P}<0.05$, when compared with young female subjects; ${ }^{*}, \mathrm{P}<0.01$, when compared with young female subjects;, $\mathrm{P}<0.01$, when compared with young male subjects. TK, thoracic kyphosis; LL, lumbar lordosis; TPA, T1 pelvic angle; SVA, sagittal vertical axis; OD-HA, odontoid with regard to hip axis.
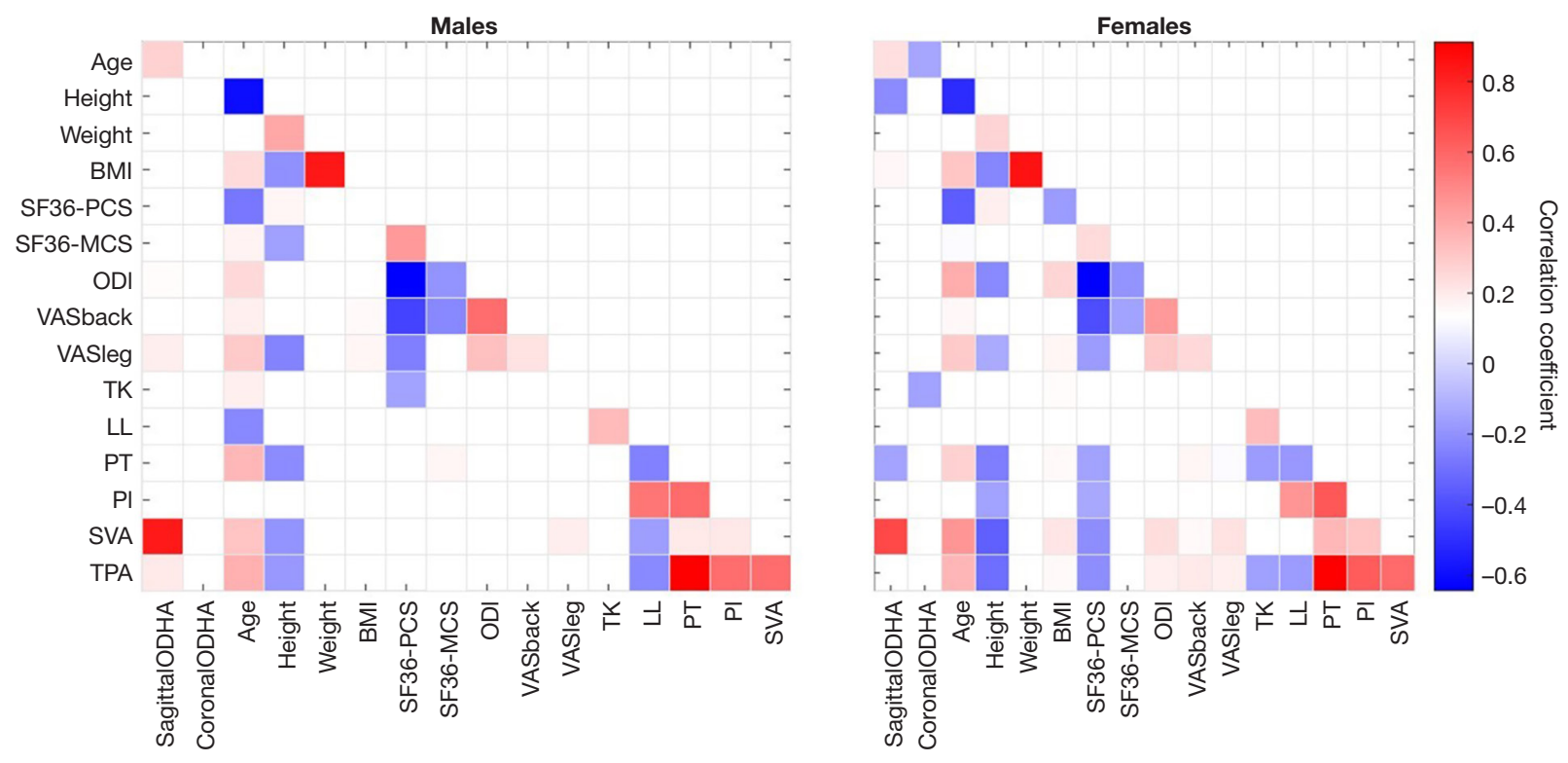

Figure 3 Correlation of spinopelvic and clinical parameters. Colors represent the correlation coefficient resulting from Pearson's or Spearman's test, according to data normality. Only those variables with significant correlations are reported (for instance, Sex was not correlated to any other parameter), and only significant correlations are reported for the remaining variables $(\mathrm{P}<0.05)$. Coronal OD-HA was reported even if was not correlated with other variables as one of the main parameters of interest for the study. OD-HA, odontoid-hip axis.

OD-HA (Figure 4). Based on the analysis, both sagittal and coronal OD-HA were related to sex, weight, TK, PT, SVA, TPA and ODI. All parameters had a positive effect on both sagittal and coronal OD-HA but PT, ODI and TPA, which had a negative effect. Age was not retained in the stepwise analysis as a determinant of OD-HA, which suggests that the correlation between OD-HA and age is indirect.

Finally, principal components analysis on these interrelated variables showed that the first three principal components explained $60 \%$ of the data variability.
Sagittal OD-HA plays a major role in all three principal components, as can be inferred by the relative length of the vectors representing OD-HA in the biplot in Figure 4. Coronal OD-HA, on the other hand, is responsible for less variability of the data. Sagittal OD-HA was mainly associated with SVA and patient weight.

\section{Discussion}

The measurement of the composite radiographic 

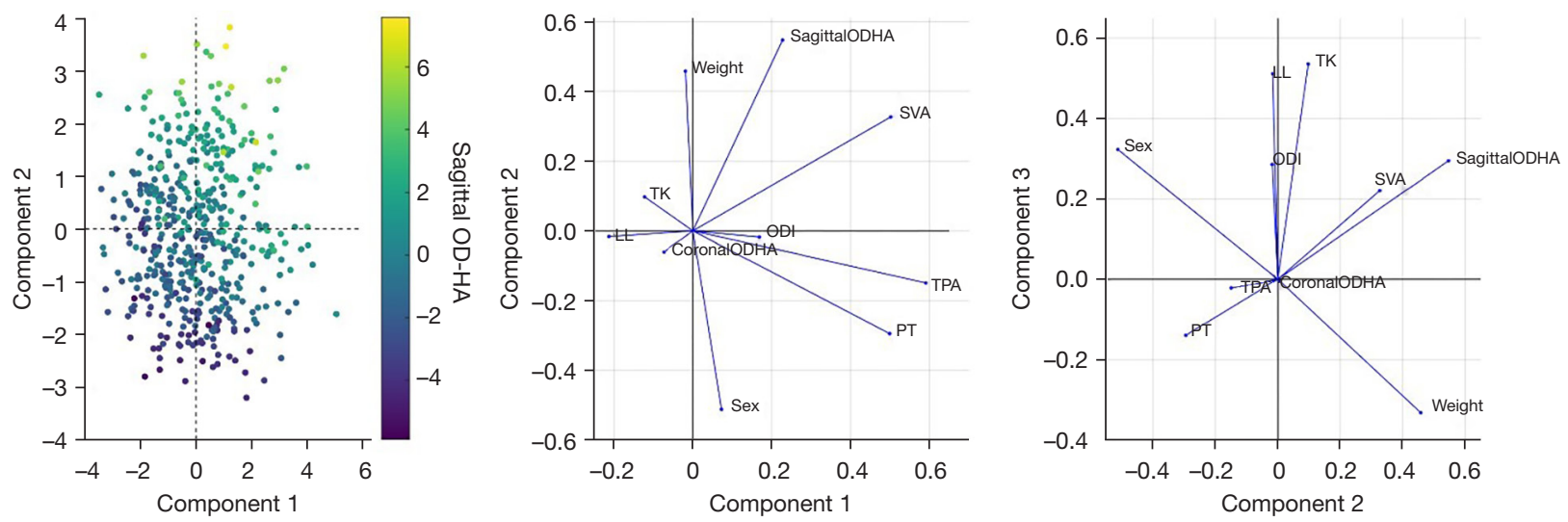

Figure 4 Principal component analysis of parameters related to sagittal and coronal OD-HA. Left panel shows the scores plot of the first two components, with the color code representing sagittal OD-HA. Right panel shows a biplot with the included variables. ODHA, odontoid-hip axis; SVA, sagittal vertical axis; TPA, T1 pelvic angle; PT, pelvic tilt; TK, thoracic kyphosis; LL, lumbar lordosis; ODI, Oswestry disability index.

parameters is essential to understand the compensatory mechanism when treating patients with spinal deformity quantitatively (23). Although numerous studies on global spinal balance in 2D radiographs has helped to advance the understanding and classification of spinal deformity, the lack of characterization of a true $3 \mathrm{D}$ multiplanar deformity remains a significant limitation (24-26). With the development and widespread availability of EOS imaging system in recent years, 3D analysis in spinal deformity research become possible (26). In this study, we conducted a pilot validation of a $3 \mathrm{D}$ global spinal balance parameter, OD-HA angle, in a large cohort with even age and gender distribution aged from 20 to 89 years old in a large Chinese adult cohort.

The results of this study revealed very little variability in the 3D OD-HA angle across the different age groups, with SD of $2.5^{\circ}$ and $1.1^{\circ}$ in sagittal and coronal planes, respectively. In Amabile's study on the asymptomatic European subjects, the results also suggested that the ODHA angle was less variable as compared with other global spinal balance parameters and more robust when it comes to digitizing the point on the radiographies (12). Based on sagittal plane, studies could use odontoid, C7 or T1 centroid to characterize uniplanar global alignment with OD-HA, SVA and TPA that appear as complementary parameters. Clinically, OD-HA angle may be more accurate when assessing the global spinal balance in both coronal and sagittal plane by taking into account the head and cervical spine, in contrast to SVA or TPA $(1,27,28)$.

Previous studies have focused on other specific points to quantify the alignment of the head with spine or pelvis, such as the McGregor line, or cranial center of mass $(29,30)$. Although all these points were close to the true center of mass of head to assess its alignment compared to the pelvis, a reliable, precise and easy-to-implement method is necessary to identify points of interest in the busy clinical setting. With these requirements, our research group had made great efforts to explore this new index, where only points directly identifiable on the $3 \mathrm{D}$ model of the spine were considered.12 The current study provided further investigation and validation on the changes of the new index with age and gender in a much bigger cohort of adults.

According to the results of this study, OD-HA showed very limited increase from $20 \mathrm{~s}$ to $80 \mathrm{~s}$ (sagittal: $-1.3^{\circ}$ to $0.8^{\circ}$ for female, $-0.4^{\circ}$ to $1.7^{\circ}$ for male; coronal: male: from $0.3^{\circ}$ to $-0.6^{\circ}$; female: $0.4^{\circ}$ to $0.2^{\circ}$ ). Correlation analysis revealed that both sagittal and coronal OD-HA had significant but mild correlation with age ( $\mathrm{r}=0.278$ and -0.157 , respectively). This suggested OD-HA angle remained stable from young to old in asymptomatic population and confirmed the hypothesis that the head tends to remain above the pelvis in a small cone of stability physiologically. In addition, this study provided the reference range of the $3 \mathrm{D}$ global spinal balance in each decade and gender based on a large-scale asymptomatic population.

The results from this study on Chinese population was also compared with a European cohort studied with the same protocol. The results showed that the values of coronal and sagittal OD-HA angle in Chinese group were statistically significantly higher than that in the European 
group both in young and older group $(\mathrm{P}<0.05)$. The higher values of OD-HA indicate that the Chinese subjects tend to be more leaning forward in the sagittal plane as compared with the European subjects. However, the mean differences were small (less than $2^{\circ}$ ), unlikely to be of clinical significance.

Our protocol complied with Fechtenbaum's study that subjects stand in a comfortable position with hips and knees extended and with hands on a support in order to decrease the possibility of motion artifact that may cause difficulty in 3D reconstruction, whereas the Arts et Métiers ParisTech adapted Faro's method that subjects stand in the free standing position with the hands resting on the mandibles $(22,31)$. Hence, the variation on the standing position between the two populations during scanning might potentially amplify the measured differences of the OD-HA angle. Nevertheless, further investigations would be needed. In addition, we define "asymptomatic subjects" as those volunteers who have no clinical neck or back pain nor any related medical consultations or surgical treatment in the past six months. In addition to the spine, we also actively exclude subjects with pathology or surgery of the hip or knee that could affect coronal or sagittal balance.

In our recent study, we found that the subjects aged above or below 50 years engaged different compensation mechanism of sagittal alignment (32). In the current study, therefore, we conducted subgroup analysis by using the age of 50 as a cut-off to divide the cohort into young and elderly group. The results revealed that there was significant difference in OD-HA angle and SVA $(\mathrm{P}<0.05)$. It suggested that the young and elderly subjects presented with a different compensation mechanism to maintain global spinal balance, which was in accordance with our previous study.

The analysis of the correlation and determinants of OD-HA confirms that balance is a full-body issue, and that different subjects might deploy different strategies to keep the head above the pelvis; indeed, while spinopelvic parameters are often intercorrelated, such as TK, LL and PT (Figure 3), sagittal and coronal OD-HA appear independent. Results also suggest that differences are present between in males and female. The latter showed correlations between OD-HA, PT and TK (Figure 3), but not their male counterparts. Sagittal OD-HA was strongly correlated to SVA, which was expected since the two parameters reflect a similar sagittal imbalance. Surprisingly, TPA was only weakly correlated to sagittal OD-HA in males, but not in females. It is also interesting to notice that in this cohort, coronal OD-HA is responsible for less data variability than sagittal OD-HA (Figure 4). This could be expected, since aging is more often associated with sagittal imbalance rather that coronal; coronal OD-HA is more relevant in the study of scoliosis.

One limitation of this study lies in the position of the subject in the X-ray cabin. Although the purpose of the handrail was to keep the subjects to stand still, the grasp on the handrail might have altered the volunteer's natural posture by shifting forwards or backwards the upper body. As there is no optimal standing position at this stage, a careful monitoring on the subject's position in a natural and comfortable status would be needed to truly reflect a more reliable results characteristics of the subject's upright posture.

\section{Conclusions}

In summary, the 3D OD-HA angle showed little variability from young to elderly Chinese asymptomatic adults, with standard deviations of $2.5^{\circ}$ and $1.1^{\circ}$ in the sagittal and coronal planes, respectively. OD-HA angle confirms that the head tends to remain above the pelvis in a small cone of stability physiologically. This study provides the reference range of the global spinal balance in each decade and gender based on a large-scale asymptomatic population. Importantly, this study also facilitates to initiate a new area of spinal deformity research which cross from spinopelvic alignment to global spinal balance, from two-dimension to three-dimension in the near future.

\section{Acknowledgments}

The authors are grateful to the ParisTech BiomecAM chair program on subject-specific musculoskeletal modelling (with the support of ParisTech and Yves Cotrel Foundations, Société Générale, Proteor and Covea).

Funding: This work was supported by the Health and Medical Research Fund Research Fellowship Scheme 2019 (No. 05190047).

\section{Footnote}

Reporting Checklist: The authors have completed the MDAR checklist and STROBE reporting checklist. Available at https://qims.amegroups.com/article/view/10.21037/qims$21-718 / \mathrm{rc}$

Conflicts of Interest: All authors have completed the ICMJE 
uniform disclosure form (available at https://qims. amegroups.com/article/view/10.21037/qims-21-718/coif). $\mathrm{ZH}, \mathrm{CV}, \mathrm{LG}, \mathrm{GCWM}$ and WS report that they receive research support through institutions. The other authors have no conflicts of interest to declare.

Ethical Statement: The authors are accountable for all aspects of the work in ensuring that questions related to the accuracy or integrity of any part of the work are appropriately investigated and resolved. The study was conducted in accordance with the Declaration of Helsinki (as revised in 2013). This was a cross-sectional study with approval from our institutional Ethics Committee (CREC Ref. No. 2017.689). All volunteers were fully informed about the methods, purposes, and risks involved in the study protocol and signed the informed consent.

Open Access Statement: This is an Open Access article distributed in accordance with the Creative Commons Attribution-NonCommercial-NoDerivs 4.0 International License (CC BY-NC-ND 4.0), which permits the noncommercial replication and distribution of the article with the strict proviso that no changes or edits are made and the original work is properly cited (including links to both the formal publication through the relevant DOI and the license). See: https://creativecommons.org/licenses/by-nc-nd/4.0/.

\section{References}

1. Mac-Thiong JM, Roussouly P, Berthonnaud E, Guigui P. Sagittal parameters of global spinal balance: normative values from a prospective cohort of seven hundred nine Caucasian asymptomatic adults. Spine (Phila Pa 1976) 2010;35:E1193-8.

2. Dubousset J. Three-dimensional analysis of the scoliotic deformity. The Pediatric Spine: Principles Practice, 1994.

3. Blondel B, Schwab F, Ungar B, Smith J, Bridwell K, Glassman S, Shaffrey C, Farcy JP, Lafage V. Impact of magnitude and percentage of global sagittal plane correction on health-related quality of life at 2-years follow-up. Neurosurgery 2012;71:341-8; discussion 348.

4. Schwab F, Lafage V, Patel A, Farcy JP. Sagittal plane considerations and the pelvis in the adult patient. Spine (Phila Pa 1976) 2009;34:1828-33.

5. Skalli W, Lavaste F, Descrimes JL. Quantification of threedimensional vertebral rotations in scoliosis: what are the true values? Spine (Phila Pa 1976) 1995;20:546-53.

6. Glaser DA, Doan J, Newton PO. Comparison of 3-dimensional spinal reconstruction accuracy: biplanar radiographs with EOS versus computed tomography. Spine (Phila Pa 1976) 2012;37:1391-7.

7. Al-Aubaidi Z, Lebel D, Oudjhane K, Zeller R. Threedimensional imaging of the spine using the EOS system: is it reliable? A comparative study using computed tomography imaging. J Pediatr Orthop B 2013;22:409-12.

8. Green RA, Saifuddin A. Whole spine MRI in the assessment of acute vertebral body trauma. Skeletal Radiol 2004;33:129-35.

9. Dubousset J, Charpak G, Dorion I, Skalli W, Lavaste F, Deguise J, Kalifa G, Ferey S. A new 2D and 3D imaging approach to musculoskeletal physiology and pathology with low-dose radiation and the standing position: the EOS system. Bull Acad Natl Med 2005;189:287-97; discussion 297-300.

10. Dubousset J, Charpak G, Skalli W, Deguise J, Kalifa G. EOS: a new imaging system with low dose radiation in standing position for spine and bone \& joint disorders. Journal of Musculoskeletal Research 2010;13:1-12.

11. Vital JM, Senegas J. Anatomical bases of the study of the constraints to which the cervical spine is subject in the sagittal plane. A study of the center of gravity of the head. Surg Radiol Anat 1986;8:169-73.

12. Amabile C, Pillet H, Lafage V, Barrey C, Vital JM, Skalli $W$. A new quasi-invariant parameter characterizing the postural alignment of young asymptomatic adults. Eur Spine J 2016;25:3666-74.

13. Amabile C, Le Huec JC, Skalli W. Invariance of headpelvis alignment and compensatory mechanisms for asymptomatic adults older than 49 years. Eur Spine J 2018;27:458-66.

14. Vergari C, Courtois I, Ebermeyer E, Pietton R, Bouloussa H, Vialle R, Skalli W. Head to pelvis alignment of adolescent idiopathic scoliosis patients both in and out of brace. Eur Spine J 2019;28:1286-95.

15. Yeung KH, Man GCW, Skalli W, Hu Z, Hung VWY, Hung ALH, Lam TP, Ng BKW, Cheng JCY, Vergari C, Chu WCW. Global sagittal alignment after surgery of right thoracic idiopathic scoliosis in adolescents and adults with and without thoracic hypokyphosis. Sci Rep 2021;11:6294.

16. Le Huec JC, Charosky S, Barrey C, Rigal J, Aunoble S. Sagittal imbalance cascade for simple degenerative spine and consequences: algorithm of decision for appropriate treatment. Eur Spine J 2011;20 Suppl 5:699-703.

17. Benoist $M$. Natural history of the aging spine. Eur Spine J 2003;12 Suppl 2:S86-9. 
18. Alzakri A, Vergari C, Van den Abbeele M, Gille O, Skalli W, Obeid I. Global Sagittal Alignment and Proximal Junctional Kyphosis in Adolescent Idiopathic Scoliosis. Spine Deform 2019;7:236-44.

19. Vialle R, Levassor N, Rillardon L, Templier A, Skalli W, Guigui P. Radiographic analysis of the sagittal alignment and balance of the spine in asymptomatic subjects. J Bone Joint Surg Am 2005;87:260-7.

20. Mac-Thiong JM, Roussouly P, Berthonnaud E, Guigui P. Age- and sex-related variations in sagittal sacropelvic morphology and balance in asymptomatic adults. Eur Spine J 2011;20 Suppl 5:572-7.

21. Zhu Z, Xu L, Zhu F, Jiang L, Wang Z, Liu Z, Qian BP, Qiu Y. Sagittal alignment of spine and pelvis in asymptomatic adults: norms in Chinese populations. Spine (Phila Pa 1976) 2014;39:E1-6.

22. Faro FD, Marks MC, Pawelek J, Newton PO. Evaluation of a functional position for lateral radiograph acquisition in adolescent idiopathic scoliosis. Spine (Phila Pa 1976) 2004;29:2284-9.

23. Harroud A, Labelle H, Joncas J, Mac-Thiong JM. Global sagittal alignment and health-related quality of life in lumbosacral spondylolisthesis. Eur Spine J 2013;22:849-56.

24. Sullivan TB, Reighard FG, Osborn EJ, Parvaresh KC, Newton PO. Thoracic Idiopathic Scoliosis Severity Is Highly Correlated with 3D Measures of Thoracic Kyphosis. J Bone Joint Surg Am 2017;99:e54.

25. Diebo BG, Oren JH, Challier V, Lafage R, Ferrero E, Liu S, Vira S, Spiegel MA, Harris BY, Liabaud B, Henry JK, Errico TJ, Schwab FJ, Lafage V. Global sagittal axis: a step toward full-body assessment of sagittal plane deformity in the human body. J Neurosurg Spine 2016;25:494-9.

26. Protopsaltis T, Schwab F, Bronsard N, Smith JS, Klineberg E, Mundis G, Ryan DJ, Hostin R, Hart R,

Cite this article as: Hu Z, Vergari C, Gajny L, Man GCW, Yeung KH, Liu Z, Lam TP, Zhu Z, Qiu Y, Chu WCW, Cheng JCY, Skalli W. An analysis on the determinants of head to pelvic balance in a Chinese adult population. Quant Imaging Med Surg 2022;12(4):2311-2320. doi: 10.21037/qims-21-718
Burton D, Ames C, Shaffrey C, Bess S, Errico T, Lafage $\mathrm{V}$; International Spine Study Group. TheT1 pelvic angle, a novel radiographic measure of global sagittal deformity, accounts for both spinal inclination and pelvic tilt and correlates with health-related quality of life. J Bone Joint Surg Am 2014;96:1631-40.

27. Ilharreborde B, Dubousset J, Le Huec JC. Use of EOS imaging for the assessment of scoliosis deformities: application to postoperative $3 \mathrm{D}$ quantitative analysis of the trunk [published correction appears in Eur Spine J. 2014 Jul;23 Suppl 4:S468]. Eur Spine J 2014;23 Suppl 4:S397-405.

28. Roussouly P, Pinheiro-Franco JL. Sagittal parameters of the spine: biomechanical approach. Eur Spine J 2011;20 Suppl 5:578-85.

29. Le Huec JC, Demezon H, Aunoble S. Sagittal parameters of global cervical balance using EOS imaging: normative values from a prospective cohort of asymptomatic volunteers. Eur Spine J 2015;24:63-71.

30. Sugrue PA, McClendon J Jr, Smith TR, Halpin RJ, Nasr FF, O'Shaughnessy BA, Koski TR. Redefining global spinal balance: normative values of cranial center of mass from a prospective cohort of asymptomatic individuals. Spine (Phila Pa 1976) 2013;38:484-9.

31. Fechtenbaum J, Etcheto A, Kolta S, Feydy A, Roux C, Briot K. Sagittal balance of the spine in patients with osteoporotic vertebral fractures. Osteoporos Int 2016;27:559-67.

32. Hu Z, Man GCW, Yeung KH, Cheung WH, Chu WCW, Law SW, Lam TP, Zhu Z, Qiu Y, Cheng JCY. 2020 Young Investigator Award Winner: Age- and Sex-related Normative Value of Whole-body Sagittal Alignment Based on 584 Asymptomatic Chinese Adult Population From Age 20 to 89. Spine (Phila Pa 1976) 2020;45:79-87. 